\title{
Efficacy of BCG vaccine and role of non-specific sensitivity due to atypical mycobacteria
}

\author{
Rajan R Patil ${ }^{1 *}$ and Krishnamurthy $\mathrm{MS}^{2}$ \\ ${ }^{1}$ School of Public Health, SRM University, Chennai \\ ${ }^{2}$ National Tuberculosis Institute, Bangalore (Retired)
}

\begin{abstract}
This paper attempts to summarizes possible reasons for BCG trial failure in India. It also lists out some of the important controversies and questions raised with regards to BCG trial in the context of Non-Specific sensitivity. It emphasizes on the fact, that Tuberculin test does not accurately reflect individuals responded immunologically to mycobacterium, it only indicates nature of immune response that has been elicited in an infected individual. The dynamics of tuberculin reaction needs to be understood through immunological considerations. Categorization of Tuberculin test results factoring in immunological considerations, will have great implication on the interpretation of tuberculin test and therefore on the estimation of annual risk of infection of tuberculosis.
\end{abstract}

\section{Background}

Failure of BCG vaccine to protection in India against pulmonary tuberculosis gave rise plethora of controversy over the design and conduct of the study [1]. Most of the controversies surrounding BCG trial has been long settled. However there still lingering questions over the possibility of role played by Non-Specific Sensitivity[NSS] caused through environmental atypical mycobacterium infection possibly masking the protective effect of BCG in the trial area continue to be debated $[2,3]$

To give the readers the context of NSS and the controversies surrounded, a brief overview of BCG trial in India is presented below.

Rationale behind carrying out BCG randomize control trial in India were [4]:

1. Emergence of contradictory findings on efficacy of BCG from developed countries (UK- 80\% BMRC trial, USA- 0-33\% (source IJMR 79-80)

2. All earlier controlled human trials were conducted with hash liquid vaccines and not freeze-dried vaccine that was extensively being used tuberculosis control programme in various countries.

3. Expert group that reviewed these BCG trials carried outside India opined that the differences in efficacy of BCG was probably due to [5]:

I. Dose and strength of the vaccine

II. Prevalence of NSS infection in certain areas and this infection showing protection in animal experiment.

III. Probably due to usage of liquid/freezed and dried vaccine.

IV. Logistics and operational reasons like method of administration, cold Chain management, training, standardization of technique.

BCG trial in India was designed taking care of all the above identified factors responsible for influencing the efficacy of vaccine adversely

\section{Design and Outcome of BCG Trial in India}

This BCG trial was organised by the Indian Council of Medical Research (ICMR) in collaboration with the WHO and Centres for Disease Control (CDC), US Public Health Services. The objective of the study was to estimate of the protective effect of BCG vaccination against Tuberculosis. The study had sample size about 2,60,000. Follow up was sequential in 6 intervals of 2.5 years each, for a total of 15 years. Among a total of 281,161 persons aged 1 month or above, two-thirds were given BCG and a third were given placebo in randomized blind allocation. In the pre-vaccination stage, everyone beyond infancy was tested with Purified Protein Derivatives PPD-S and PPD-B (mycobacterium of Battey strain of $\mathrm{M}$. avium, representing environmental non-TB mycobacterium). Also, everyone above 10 years was X-rayed. Those below 10 were assumed to be free from $\mathrm{TB}$, unless ill. The subsets of population analyzed for BCG efficacy were those with $<8 \mathrm{~mm}$ PPD-S reaction and normal chest $\mathrm{X}$-ray at recruitment and those who were infants at that time. They were defined as uninfected by Mycobacterium tuberculosis at recruitment [6]. The results of $71 / 2$ years and 15year follow up showed that the number of new cases that occurred among the group vaccinated by either of the vaccines or from the unvaccinated group were similar. This showed that BCG vaccination did not offer any protection against tuberculosis of the lung [7].

An expert group was constituted in 1980s to review and Analyze the data from South Indian BCG trial [8], and provide the probable explanations that could be attributed for the failure of BCG Vaccine in India. The reasons attributed were:

Correspondence to: Rajan R Patil, Division of Epidemiology, School of Public Health, SRM University, Potheri, Kattankulathur-603203, Greater Chennai, India, Tel: 91 9445811610; E-mail: rajanpatil@yahoo.com

Key words: BCG vaccine, efficacy, non-specific sensitivity, tuberculin

Received: November 22, 2017; Accepted: December 23, 2017; Published: December 26, 2017 
1. High prevalence of NSS infection in the study area masking the protective value of BCG

2. Higher Virulence of the Bacelli prevalent in the study area

3. The TB epidemiological situation in the study area.

However, the expert committee recommended the continued usage of BCG in children below one-year age based on the available indirect evidences that it offers protection against development of childhood form of tuberculosis.

\section{Discussion}

Divergent interpretation of role of NSS in the trial area by different investigators- had lead to confusion.

In the trial area the researchers could classify the study subjects based on interpretation of tuberculin test and PPD-B antigen. The efficacy was estimated based on PPD-S Non-reactors only between vaccinated and control group.

I. The role of NSS in epidemiology of tuberculosis is highly controversial. While few researchers have argued that that such sensitivity may offer protection against the development TB [2,3] but other researchers have provided counter evidence.

In 2006, Tuberculosis Research Centre (TRC) Madras re-analyzed the data of NSS infection based on Age, Sex and its influence on TB incidence. The subjects were classified based on PPDS and PPDB as reactors and non-reactors for NSS. It was again concluded that both in children as well as adult age group, NSS has not played any role in prevention of TB [11].

II. Re-examining the same data analysed by TRC Madras, Grahm etal have reported that PPD B does have protective effect up to $37 \%$ by examining PPD -ve group against PPD +ve group among placebo group [12].

III. Also, Grahm et all commenting on the reanalysis made by TRC in 2006 have concluded that in those study subjects who had been classified as Non-Reactors for PPD-B and PPD-S, had overall the protection of $29 \%$ (and $37 \%$ if only high dose was considered) was observed, among those who were vaccinated initially irrespective of the strength of the vaccine given. Therefore, from TRC data -they have concluded that NSS has a role to play in offering protection among vaccinated with following conclusion

a) Exposure to environmental mycobacteria that results in skin test responsiveness to PPD-B correlates with a degree of protection from $\mathrm{TB}$

b) Subsequent BCG vaccination does not boost this protection; and

c) BCG can evoke similar protection in people who are "mycobacterially naïve".

They have also supported the hypothesis that BCG vaccination does offers protection against childhood form of TB.

\section{BCG- NSS interaction}

The role of NSS infection in masking BCG protective effect against development of tuberculosis have been based on the following premise

1. Animal experiments have shown NSS offer protection against development of disease in guinea pigs.

2. Vaccination failure was attributed for prevalence of NSS in America.
3. Analysis of the data for of NSS classifying the subjects based on PPD -B, PPD-B\& PPD-S reaction have reported that PPD-B has no role to play in development of TB.

4. Indian BCG trial showed the PPD-B negative, PPD-S negative individuals form a minority of the population, and a protection rate of 37 per cent in only 40 per cent of the community [12]. While many researchers accept some protective effect by NSS but doubt the clinical importance of this effect is unlikely to impact on over all TB epidemiology and tuberculosis control in the community.

It is important to note here that emergence of such data has taken nearly 3 decades to evolve and reach present conclusion. The latest understanding is that the tuberculin test does not accurately reflect whether an individual has encountered or responded immunologically to mycobacteria.

Taking into consideration Immunological dynamics the Tuberculin test results would now need to be classified in three groups namely:

1. Tuberculin non-reactors.

2. Tuberculin non-reactors but immunologically active

3. Tuberculin reactors probably immunologically active

\section{Public health implications}

Categorization of Tuberculin test results taking into immunological considerations will have great implication on the interpretation of tuberculin test and further on the estimation of annual risk of infection. This would imply:

1. Hypersensitivity does not represent immunity of TB. Hence two are different.

2. Since the basis of ARI is hypersensitivity to tuberculin test- ARI survey results then provide underestimate tuberculosis burden.

3. BCG has no role to play in public health prevention of Tuberculosis in the community, except in vergin islands where TB infection is beginning to get foothold. Even in such areas BCG will help only in early stages of TB epidemiology, once the tuberculosis gets firmly established in such areas- BCG again looses its public health utility as it no more helps in TB control in the community [13].

4. Tuberculin test negative results cannot be justification for complacence of absence of TB infection, unless immunogenic tests are carried out concurrently

\section{Conclusions \& future direction for research in NSS and $\mathrm{TB}$}

1. Experimental studies need to be carried out on the group of people who have been exposed totally to open cases of tuberculosis

2. Such exposed group need to be subjected to dual investigation namely tuberculin test and immunological test.

3. Classify Such exposed group based on Tuberculin and Immunological results as

a) Tuberculin non-reactors.

b) Tuberculin non-reactors but immunologically active

c) Tubercluin reactors probably immunologically active

4. Correction factor need to be worked out for revising the estimate of ARI. 
5. By observing the incidence rates among these three groups the most risk groups in this can be identified for taking needful intervention measure.

6. The classification of NSS group has till now been done using solely PPD-B only. However, if species specific antigen to be used for assessing the prevalence of different types of NSS, findings could have been more interesting than what has been reported.

\section{References}

1. (1980) Tuberculosis Prevention Trial, Madras, Trial of BCG vaccines in south India for tuberculosis prevention. Ind J Med Res 72: 1

2. Palmer C.E \& Long MW (1966) American review of respiratory diseases 94: 553-568

3. Raj Narain (1972) American review of Respiratory diseases 105: 578-585.

4. (1980) Tuberculosis Prevention Trial, Madras. Trial of BCG vaccines in south India for tuberculosis prevention. Ind J Med Res 72: 1.

5. (1980) WHO Technical Report Series (Report of WHO Scientific Group on Vaccination against Tuberculosis)
6. John TJ (2000) Tuberculosis control without protec-tion from BCG. Indian Pediatr 37: $9-18$

7. (1999) Tuberculosis Research Centre. Fifteen year follow-up of trial of BCG vaccines in south India for tuberculosis prevention, Ind.J.Med.Res 110: 56.

8. (1979) Tubecuolosis Prevention trial. Trial of BCG Vaccines in south India for tuberculis prevention : frist report. Bulletin WHO 57: 819-827

9. (1967) D’ Aacry Hart,P. British Medical Journal 1: 587-592.

10. (1974) Comstock GW. AJPH 64:3

11. (2006) Tuberculosis Research Centre (ICMR), Chennai. Influence of sex, age and nontuberculous infection at intake on the efficacy of BCG: re-analysis of 15-year data from a doubleblind randomized control trial in south India. Indian J Med Res 123: 119-24.

12. Rook G A.W. \& Kim L U (2006) Comments on the new analysis of the Chingleput BCG trial Indian. J Med Res 123: 103-106.

13. Chakraborty AK, Channabasavaiah R, Krishnamurthy MS, Shashidhara AN, Motiram G (1991) Tuberculin skin sensitivity following chemoprophylaxis in an island community. Indian J Tuberc 38: $201-211$.

Copyright: @2017 Patil RR. This is an open-access article distributed under the terms of the Creative Commons Attribution License, which permits unrestricted use, distribution, and reproduction in any medium, provided the original author and source are credited. 